\title{
Regulatory B cells in autoimmune rheumatic diseases
}

\section{Lazaros I. Sakkas}

Mediterr J Rheumatol 2017; 28(2):75-9

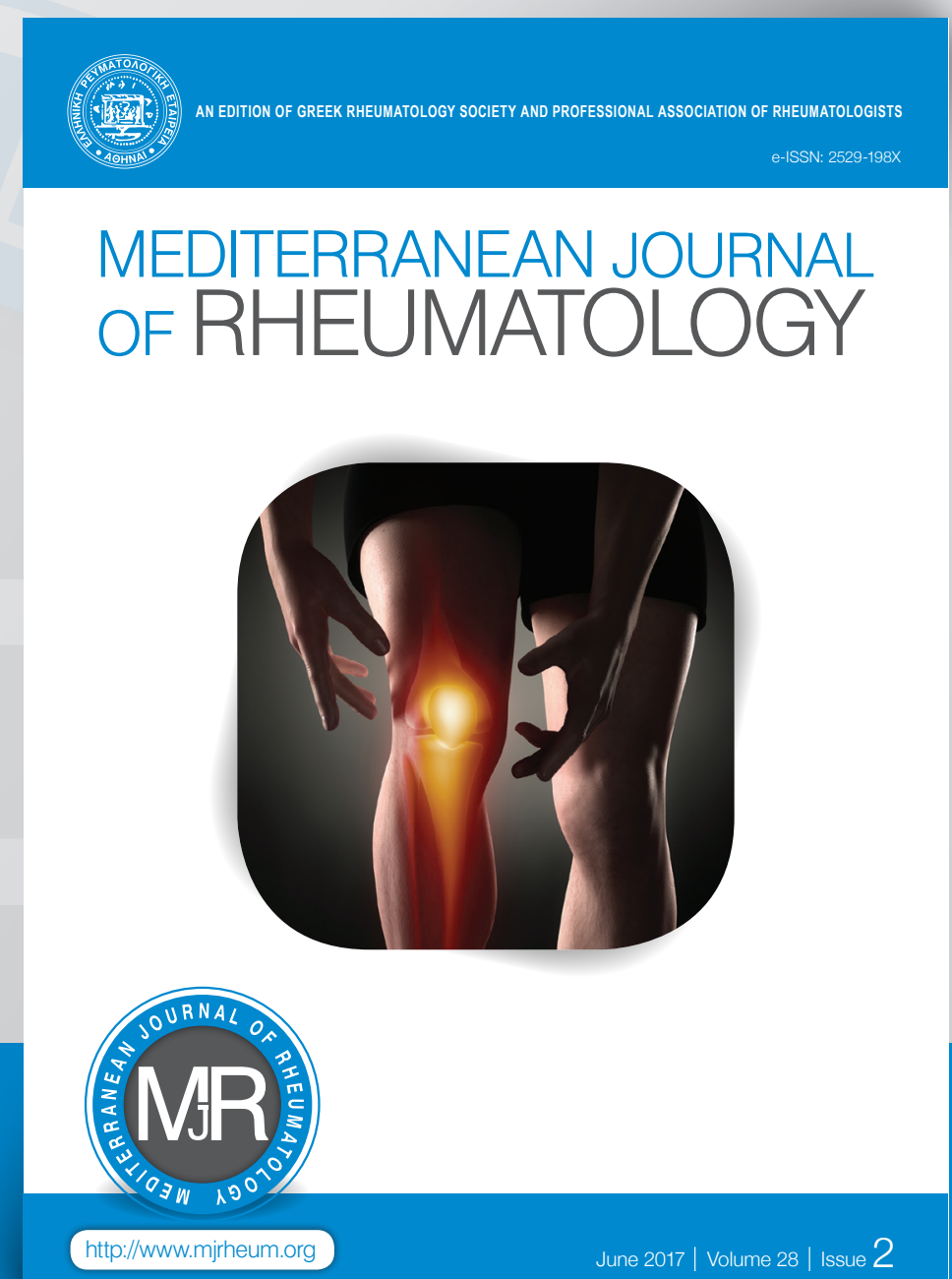


๑) Sakkas LI

\section{Regulatory B cells in autoimmune rheumatic diseases}

\section{Lazaros I. Sakkas}

Department of Rheumatology and Clinical Immunology, Faculty of Medicine, School of Health Sciences, University of Thessaly, Larissa, Greece

\section{ABSTRACT}

Background: Regulatory B cells (regulatory B cells, Breg cells) in recent years have been shown to be important immunoregulatory factors. Aim: To review the role of Breg cells in autoimmune rheumatic diseases. Methods: This descriptional review was carried out after research on PubMed using the keywords "Bregs and rheumatoid arthritis", "systemic lupus erythematosus", "Sjögren's syndrome", "systemic sclerosis", "vasculitis", and "dermatomyositis". Results: Breg cells have an inhibitory effect on pro-inflammatory Th1 and Th17 cells and prevent the development of autoimmune diseases. Breg cells mediate their effects through interleukin-10 (IL-10, IL-10+Breg cells), but recently other Breg cells have been recognized that mediate their effects through IL-35 (IL-35+Breg cells), or through transforming growth factor- $\beta$ (TGF $\beta$, TGF $\beta+$ Breg cells). In experimental models of autoimmune diseases, Breg cells are decreased, and when expanded ex vivo and re-infused back into animals, they ameliorate disease. In humans, IL-10+Breg cells are decreased in active autoimmune diseases, such as rheumatoid arthritis, ANCA-associated vasculitis, and systemic sclerosis, and may increase to normal levels in disease remission. Conclusions: The deficiency of IL-10+Breg cells during active autoimmune rheumatic disease suggests that Breg cells may be used as biomarkers and be a possible therapeutic target in these diseases.

Mediterr J Rheumatol 2017;28(2):75-9

https://doi.org/10.31138/mjr.28.2.75

Article Submitted 27/12/2016, Revised Form 14/02/2017, Accepted 25/02/2017

Keywords: Breg cells, rheumatoid arthritis, systemic lupus erythematosus, systemic sclerosis, vasculitis, dermatomyositis.

\section{INTRODUCTION}

The adaptive immune system, in order to restrict the immune response to a pathogenic agent and prevent autoimmunity is equipped

Corresponding author: Lazaros I. Sakkas, MD, DM, PhD(UK), $\mathrm{FRCP}(\mathrm{UK})$

Department of Rheumatology and Clinical Immunology University of Thessaly, Faculty of Medicine, School of Health Sciences

41110 Larissa, Greece

Tel.: +302413502813

Fax: +302413501016

E-mail: Isakkas@med.uth.gr the regulatory $\mathrm{T}$ cells (Treg cells). During the last 15 years it has been shown that a subset of $B$ cells also exhibits immunoreg- regulatory $B$ cells (Breg cells) This study is a descriptive review. The author searched the PubMed using the keywords "Breg cells" and "rheumatoid arthritis", "systemic lupus erythematosus", "Sjögren's syndrome”, "systemic sclerosis", "dermatomyositis", and "vasculitis". An extensive general bibliography for Breg cells is also at the author's disposal. ulatory functions, the

\section{REGULATORY B CELLS}

Breg cells, although a small proportion of peripheral blood B cells, play a major role in controlling the immune response and preventing autoimmunity. Breg cells that 
have been studied most thoroughly are those that produce interleukin-10 (IL-10, IL-10+Breg cells, B10 cells). B10 cells, through IL-10 production, inhibit Th1 and Th17 cells and sustain/enhance Tregs and ameliorate experimental arthritis. ${ }^{1}$ B10 cells also decrease activation of macrophages and dendritic cells and their tumor necrosis factor-a (TNFa) production ${ }^{2,3}$ and the antigen-presenting capacity of dendritic cells, ${ }^{4}$ thus decreasing proliferation of T cells (Figure 1). The effect of Breg cells on T cells is mediated via IL-10, cell-to-cell contact through CTLA4, ${ }^{5} \mathrm{IL}-21$ receptor, CD40, and MHC-class $11 .{ }^{6}$ For example, B cells deficient in MHC-class II and B7 do not inhibit T cells. ${ }^{7}$

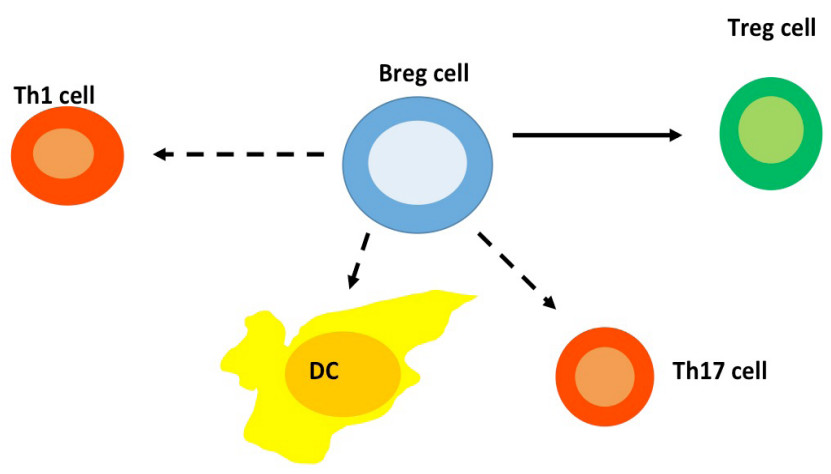

Figure 1: Regulatory B cells (Breg cells) inhibit proinflammatory Th1 and Th17 cells decrease the antigenpresenting capacity of dendritic cells (DCs) and sustain/ expand regulatory T cells (Treg cells).

However, there are other Breg cells that mediate their suppressive effect through IL-35 production (IL-35+Bregs) or through transforming growth factor- $\beta$ production (TGF $\beta$, TGF $\beta+$ Breg cells), but also through expression of other molecules, such as Foxp3 (Foxp3+CD19+CD5+Breg cells). ${ }^{8,9} \mathrm{IL}-35$ is a heterodimer comprised of IL-12p35 and the product of Epstein-Bar virus-induced gene 3 (EBi3, IL-12p35/EBi3) and IL-35+Breg cells expanded with IL-35 decrease the activation and the antigen-presenting capacity of $B$ cells. ${ }^{10} \mathrm{~B}$ cell-derived TGF $\beta 1$ inhibits Th1 and Th17 immune responses through decrease of antigen-presenting capacity of dendritic cells in experimental autoimmune encephalomyelitis (EAE). ${ }^{11}$

In humans, B10 cells of peripheral blood are increased in childhood (8-10 years-old) and are low in adults. ${ }^{12}$ Interestingly, B10 cells are very low in advanced age where they exhibit a negative correlation with serum levels of rheumatoid factor; ${ }^{13}$ a finding that is in line with the concept that deficiency of Breg cells likely contributes to loss of immunological tolerance and the development of autoimmunity. Breg cells are very low in peripheral blood and have to be increased in order to be studied. In the laboratory, classic stimuli for the detection of intracellular cytokines are phorbol 12-myristate 13-acetate (PMA) plus ionomycin which give a frequency of Breg cells $<1 \%$ of peripheral blood B cells. Stimulation of B10 progenitor cells for more expansion of B10 cells requires stimulation with $\mathrm{CpG}$ or liposaccharide (LPS) or CD40. ${ }^{3}$ In mice, B10 cells for their expansion require CD40 and IL-21receptor signaling from $\mathrm{T}$ cells. ${ }^{6} \mathrm{~A}$ proliferation inducing ligand (APRIL) induces IL-10 production in CpG-stimulated B cells, ${ }^{14}$ whereas LPS or anti-IgM stimulation induces Foxp3 expression in B cells. ${ }^{15}$

Many efforts have been made in order to identify Breg cells by cell surface markers, a requirement for functional assays. In addition, this makes their identification easy by flow cytometry. B10 cells are mainly within CD19+ CD24highCD38high (transitional Breg cells) and CD19+ CD24highCD27+ (memory Breg cells). 3,16,17 However, other markers have also been described for B10 cells, including CD19+CD1dhighCD5+, ${ }^{18}$ and CD19+Tim-1+ (T cell immunoglobulin mucin domain-1). ${ }^{19}$ One study reported that IL-10-producing Breg cells are mainly within the CD19+ CD25high population and that their regulatory effect on Treg cells was dependent on direct Breg and Treg cells contact. $^{20}$

\section{RHEUMATOID ARTHRITIS}

In most studies, B10 cells are found to be decreased in rheumatoid arthritis (RA) compared to healthy controls. ${ }^{17,21-23}$ CD19+CD24highCD38high Breg cells were found to be decreased in active RA relative to inactive RA and healthy controls and did not have the ability to inhibit Th17 cells and expand Treg cells. ${ }^{17}$ In another study, the percentages of CD24highCD38high and CD24highCD27+ Breg cells were similar to those of healthy controls, but B10 cells were decreased in RA and had an inverse correlation with disease activity (DAS28), serum levels of C-reactive protein (CRP) and serum levels of rheumatoid factor. ${ }^{21}$ Also, CD19+CD5+CD1dhigh Breg cells were decreased in RA and correlated inversely with DAS28. ${ }^{24}$ Finally, a study reported that the percentage of B10 cells did not differ from healthy controls, but exhibited an inverse correlation with DAS28. ${ }^{25}$

\section{SYSTEMIC LUPUS ERYTHEMATOSUS}

In systemic lupus erythematosus (SLE), CD19+ CD24highCD38high Breg cells were not decreased but produced less IL-10 compared to healthy controls and could not inhibit Th1 cells. ${ }^{16}$ In another study, CD19+ CD24highCD27+Breg cells and IL-10+CD19+Breg cells were decreased in SLE. Interestingly, CD19+ CD24highCD27+Breg cells had an inverse correlation with disease activity index (SLEDAl). ${ }^{26}$ In SLE it seems that plasmacytoid dendritic cells (pDCs) fail to induce the differentiation of CD24highCD38high B cells into IL-10+Breg 
cells. $^{27}$ In another study, the percentage of CD19+CD24highCD38high Breg cells did not differ from that of healthy controls, but IL-10+Breg cells were decreased in patients, particularly in those with nephritis. ${ }^{28}$ Breg cells that resulted after activation of B cells with anti-lgM/lgG antibodies in SLE exhibited reduced ability to inhibit $T$ cells. ${ }^{29}$ However, other studies showed that Breg cells did not have a defect in SLE. CD5+CD1dhigh as well as CD19+CD24highCD38high Breg cells were increased and produced IL-10. ${ }^{30}$ The percentage of CD25highFoxp3high Breg cells that produce IL-10 were increased in SLE and correlated with disease activity. ${ }^{31}$

In SLE, plasmacytoid dendritic cells (pDCs) through interferon-a (IFNa) do not induce differentiation of B cells into Breg cells; a differentiation that occurs in healthy individuals. However, a similar defect also occurs in B cells from healthy individuals exposed to high concentrations of IFNa, suggesting that the disturbance in SLE resides in the interaction between Breg cells and pDCs. ${ }^{27}$

In mice models of SLE, IL-10+Breg cells appear to have a protective role. ${ }^{16,32,33}$ For example, CD5+CD1dhigh Breg cells from wild-type mice transferred to CD5+CD1dhigh Breg cells-deficient mice with SLE, significantly improved the survival of these mice..$^{32}$ In addition, administration of IL-35 to MRL/lpr mice improved clinical, laboratory, and pathological lupus nephritis, and lupus disease activity, and increased IL-10+Breg cells. ${ }^{34}$

\section{SJÖGREN'S SYNDROME}

In Sjögren's syndrome, the percentage of CD19+ CD24highCD38high Breg cells was increased in active and inactive disease. ${ }^{35,36}$ However, these cells were defective, since they failed to inhibit IFN $\gamma$ production by $\mathrm{T}$ cells. $^{36}$

\section{VASCULITIDES}

Breg cells were mostly studied in ANCA-associated vasculitis, and found to be decreased. ${ }^{37-39}$ Percentages of IL-10+Breg cells and CD5+CD24highCD38high Breg cells were decreased in active ANCA-associated vasculitis and returned to normal in disease remission with concomitant decrease in serum ANCA levels. ${ }^{39}$ In another study, CD5+CD19+ B cells were decreased in active disease, returned to normal during disease remission, and decreased before disease relapse. ${ }^{37}$ These findings suggest that IL-10+Breg cells could be used as biomarkers in ANCA-associated vasculitis. However, two studies reported that in ANCA-associated vasculitis there is numerical but not functional impairment of CD19+CD24highCD27+ Breg cells. ${ }^{40,41}$

In Henoch-Schönlein vasculitis, one study found that the number of IL-10+Breg cells was lower in patients with nephritis, and that IL-10+Breg cells correlated inversely with 24-hour urine protein. ${ }^{42}$

In giant cell arteritis and polymyalgia rheumatica, one study showed that percentages of IL-10+Breg cells were within normal levels. ${ }^{43}$

\section{DERMATOMYOSITIS}

In dermatomyositis there was a decrease in CD19+ CD24highCD38high Breg cells, particularly in patients with interstitial lung disease and in patients with disease-specific autoantibodies. ${ }^{44}$

\section{SYSTEMIC SCLEROSIS}

Many lines of evidence suggest that $T$ cells and B cells are implicated in the pathogenesis of systemic sclerosis (SSc). ${ }^{45,46}$ Recently, two studies reported that Breg cells are decreased in systemic sclerosis (SSC). CD19+ CD24highCD38high transitional Bregs and CD19+ CD24highCD27+ memory Breg cells were decreased and impaired in their production of IL-10, particularly in SSc-associated interstitial lung disease. ${ }^{47}$ There was a decrease in STAT3 and p38MAPK signaling in B cells. ${ }^{47}$ Interestingly, Breg cells showed an inverse correlation with disease-specific autoantibodies, anti-topoisomerase I and anticentromere antibodies. ${ }^{48}$

\section{CONCLUSIONS AND FUTURE PERSPECTIVES}

In recent years, Breg cells have been found to represent significant immunoregulatory cells that suppress inflammatory immune responses and prevent autoimmunity. In autoimmune rheumatic diseases, IL-10+Breg cells are decreased and may return to normal during remission. In addition, they may decrease prior to disease flare. Therefore, Breg cells may be used as a biomarker, and be an attractive therapeutic target in these diseases. For instance, ex vivo expansion of B10 cells with CD40 and IL-21 receptor signaling and re-infusion into mice with established EAE ameliorates disease symptoms. ${ }^{6}$ However, in EAE, B10 cells suppress disease onset whereas Treg cells suppress long-standing disease. ${ }^{4}$ In humans, B10 cells may be resistant to killing by anti-CD20 monoclonal antibody (rituximab). ${ }^{2}$

Cells that may give large percentages of B10 cells with IL-35 and IL-21 stimulation include bone marrow, umbilical cord blood, and fat-derived mesenchymal stem cells. Bone marrow cells, stimulated with toll-like receptor 9 (TLR9) result in large percentages of CpGproB cells that differentiate into B10 cells and inhibit EAE in mice. ${ }^{49}$ In umbilical cord blood, there are increased percentages of B10 cells. ${ }^{5}$ In addition, fat-derived mesenchymal stem cells expand B10 cells and could be used therapeutically, as has been successfully applied to a mouse model of SLE. ${ }^{50}$

\section{CONFLICT OF INTEREST}

The author declares no conflict of interest. 


\section{REFERENCES}

1. Mauri C, Gray D, Mushtaq N, Londei M. Prevention of arthritis by interleukin 10-producing B cells. J Exp Med 2003;197:489-501.

2. Horikawa M, Minard-Colin V, Matsushita T, Tedder T F. Regulatory $\mathrm{B}$ cell production of IL-10 inhibits lymphoma depletion during CD20 immunotherapy in mice. J Clin Invest 2011;121:4268-80.

3. Iwata Y, Matsushita T, Horikawa M, Dilillo DJ, Yanaba K, Ventur $\mathrm{G} \mathrm{M}$, et al. Characterization of a rare IL10-competent B cell-cell subset in humans that parallels mouse regulatory B10 cells. Blood 2011 Jan 13;117:530-41.

4. Matsushita T, Horikawa M, Iwata Y, Tedder T F. Regulatory B cells (B10 cells) and regulatory $T$ cells have independent roles in controlling experimental autoimmune encephalomyelitis initiation and late-phase immuopathogenesis. J Immunol 2010;185:2240-52.

5. Sarvaria A, Basar R, Mehta R S, Shaim H, Muftuoglu M, Khoder A et al. IL-10+ regulatory $B$ cells are enriched in cord blood and may protect against cGVHD after cord blood transplantation. Blood 2016:128:1346-61.

6. Yoshizaki A, Miyagaki T, DiLillo D J, Matsushita T, Horikawa M, Kountikov E I, et al. Regulatory B cells control T-cell autoimmunity through IL-21-dependent cognate interactions. Nature 2012;491:264-8.

7. Rosser E C, Mauri C. Regulatory B cells: Origin, phenotype, and function. Immunity 2015;42:607-12.

8. Noh J, Choi W S, Noh G, Lee J H. Presence of Foxp3-expressing CD19(+)CD5(+) B Cells in Human Peripheral Blood Mononuclear Cells: Human CD19(+)CD5(+)Foxp3(+) Regulatory B Cell (Breg). Immune Netw 2010;10:247-9.

9. Ray A, Wang L, Dittel B N. IL-10-independent regulatory B-cell subsets and mechanisms of action. Int Immunol 2015;27:531-6.

10. Shen P, Roch T, Lampropoulou V, O'Connor RA, Stervbo U, Hilgenberg $E$, et al. IL-35-producing $B$ cells are critical regulators of immunity during autoimmune and infectious diseases. Nature 2014;507:366-70

11. Bjarnadottir K, Benkhoucha M, Merkler D, Weber M S, Rayne N $L$, Bernard C C, et al. B cell-derived transforming growth factor- $\beta 1$ expression limits the induction phase of autoimmune neuroinflammation. Sci Rep 2016;6:34594.

12. Kalampokis I, Venturi G M, Poe J C, Dvergsten J A, Sleasman J W, Tedder T F. The regulatory B cell compartment expands transiently during childhood and is contracted in children with autoimmunity. Arthritis Rheumatol 2017;184:48-53. https://doi.org/10.1002/ art.39820

13. Duggal N A, Upton J, Phillips A C, Sapey E, Lord J M. An age-related numerical and functional deficit in CD19(+) CD24(hi) CD38(hi) $B$ cells is associated with an increase in systemic autoimmunity. Aging Cell 2013;12:873-81.

14. Hua C, Audo R, Yeremenko N, Baeten D, Hahne M, Combe B, et al. A proliferation inducing ligand (APRIL) promotes IL-10 production and regulatory functions of human B cells. J Autoimmun 2016:73:64-72.

15. Park M K, Jung $Y \mathrm{O}$, Lee $S Y$, Lee $S$ H, Heo $Y$ J, Kim E K, et al. Amelioration of autoimmune arthritis by adoptive transfer of Foxp3-expressing regulatory $B$ cells is associated with Treg/Th17 cell balance. J Transl Med 2016;14:191.

16. Blair $P$ A, Noreña $L Y$, Flores-Borja F, Rawlings D J, Isenberg D A, Ehrenstein M R, et al. CD19(+)CD24(hi)CD38(hi) B cells exhibit regulatory capacity in healthy individuals but are functionally impaired in systemic Lupus Erythematosus patients. Immunity 2010;32:129-40.

17. Flores-Borja F, Bosma A, Ng D, Reddy V, Ehrenstein M R, Isenberg D A, et al. CD19+CD24hiCD38hi B cells maintain regulatory $\mathrm{T}$ cells while limiting $\mathrm{TH} 1$ and $\mathrm{TH} 17$ differentiation. Sci Transl Med 2013;5:173ra23.

18. Jeong $Y \mathrm{I}$, Hong $\mathrm{S} \mathrm{H}$, Cho $\mathrm{S} \mathrm{H}$, Lee W J, Lee SE. Induction of IL-10-producing CD1dhighCD5+ regulatory B cells following Babesia microti-infection. PLoS One 2012;7:e46553.

19. Ding Q, Yeung M, Camirand G, Zeng Q, Akiba H, Yagita $H$, et al Regulatory $B$ cells are identified by expression of TIM-1 and can be induced through TIM-1 ligation to promote tolerance in mice. J Clin Invest 2011;121:3645-56.

20. Kessel A, Haj T, Peri R, Snir A, Melamed D, Sabo E, et al. Human CD19+CD25high $B$ regulatory cells suppress proliferation of CD4+ T cells and enhance Foxp3 and CTLA-4 expression in T-regulatory cells. Autoimmun Rev 2012;11:670-7.

21. Daien C I, Gailhac S, Mura T, Audo R, Combe B, Hahne M, et al. Regulatory B10 cells are decreased in patients with rheumatoid arthritis and are inversely correlated with disease activity. Arthritis Rheumatol 2014;66:2037-46.

22. Ma L, Liu B, Jiang $Z$, Jiang $Y$. Reduced numbers of regulatory $B$ cells are negatively correlated with disease activity in patients with new-onset rheumatoid arthritis. Clin Rheumatol 2014;33:187-95.

23. Guo Y, Zhang X, Qin M, Wang X. Changes in peripheral CD19(+) Foxp3(+) and CD19(+)TGFb(+) regulatory b cell populations in rheumatoid arthritis patients with interstitial lung disease. J Thorac Dis 2015;7:471-7.

24. Cui D, Zhang L, Chen J, Zhu M, Hou L, Chen B, et al. Changes in regulatory $b$ cells and their relationship with rheumatoid arthritis disease activity. Clin Exp Med 2015 Aug;15:285-92.

25. Kim J, Lee H J, Yoo I S, Kang S W, Lee J H. Regulatory B cells are inversely correlated with disease activity in rheumatoid arthritis. Yonsei Med J 2014:55:1354-8.

26. Jin L, Weiqian C, Lihuan Y. Peripheral CD24hi CD27+ CD19+ B cells subset as a potential biomarker in naïve systemic lupus erythematosus. Int J Rheum Dis. 2013;16(6):698-708.

27. Menon M, Blair P A, Isenberg D A, Mauri C. A Regulatory Feedback between Plasmacytoid Dendritic Cells and Regulatory $B$ Cells Is Aberrant in Systemic Lupus Erythematosus. Immunity 2016;44:683-97.

28. Heinemann K, Wilde B, Hoerning A, Tobbe B, Kribben A, Witzke O, et al. Decreased IL-10(+) regulatory B cells (Bregs) in lupus nephritis patients. Scand J Rheumatol 2016;45:312-6.

29. Gao N, Dresel J, Eckstein V, Gellert R, Störch H, Venigalla R K C, et al. Impaired suppressive capacity of activation-induced regulatory B cells in systemic lupus erythematosus. Arthritis Rheumatol 2014;66:2849-61.

30. Yang $X$, Yang J, Chu Y, Xue $Y$, Xuan D, Zheng S, et al. $T$ follicular helper cells and regulatory $B$ cell dynamics in systemic lupus erythematosus. PLoS One 2014;9:e88441.

31. Vadasz Z, Peri R, Eiza N, Slobodin G, Balbir-Gurman A, Toubi E. The Expansion of CD25 high IL-10 high FoxP3 high B Regulatory Cells Is in Association with SLE Disease Activity. J Immunol Res 2015;2015:254245

32. Watanabe $R$, Ishiura N, Nakashima H, Kuwano Y, Okochi H, Tamaki K, et al. Regulatory B cells (B10 cells) have a suppressive role in murine lupus: CD19 and B10 cell deficiency exacerbates systemic autoimmunity. J Immunol 2010;184:4801-9.

33. Scapini $P$, Lamagna $C, \mathrm{Hu} Y$, Lee K, Tang Q, DeFranco A L, et al. $B$ cell-derived IL-10 suppresses inflammatory disease in Lyn-deficient mice. Proc Natl Acad Sci U S A 2011;108(41):E823-32.

34. Cai Z, Wong C K, Dong J, Chu M, Jiao D, Kam N W, et al. Remission of systemic lupus erythematosus disease activity with regulatory cytokine interleukin (IL)-35 in Murphy Roths Large (MRL)/lpr mice. Clin Exp Immunol 2015;181:253-66.

35. Furuzawa-Carballeda J, Hernadez-Molina G, Lima G, Rivera-Vicencio Y, Ferez-Blando K, Liorente L. Peripheral regulatory cells immunophenotyping in primary Sjögren's syndrome: a cross-sectional study. Arthritis Res Ther 2013;15:R68.

36. Lin W, Jin L, Chen $\mathrm{H}$, Wu Q, Fei $Y$, Zheng W, et al. B cell subsets and dysfunction of regulatory $B$ cells in IgG4-related disease and primary Siögren's syndrome: The similarities and differences. Arthritis Res Ther. 2014;16:R118.

37. Bunch D O, McGregor J G, Khandoobhai N B, Aybar L T, Burkart $\mathrm{M} \mathrm{E}, \mathrm{Hu} Y$, et al. Decreased CD5 B cells in active ANCA vasculitis and relapse after rituximab. Clin J Am Soc Nephrol 2013:8:382-91.

38. Wilde B, Thewissen M, Damoiseaux J, Knippenberg S, Hilhorst $M$, van Paassen $P$, et al. Regulatory $B$ cells in ANCA-associated vasculitis. Ann Rheum Dis 2013:72:1416-9. 
39. Aybar L T, McGregor J G, Hogan S L, Hu Y, Mendoza C E, Brand E J, et al. Reduced CD5(+) CD24(hi) CD38(hi) and interleukin-10(+) regulatory $B$ cells in active anti-neutrophil cytoplasmic autoantibody-associated vasculitis permit increased circulating autoantibodies. Clin Exp Immunol 2015;180:178-88.

40. Lepse N, Abdulahad W H, Rutgers A, Kallenberg C G, Stegeman C A, Heeringa P. Altered B cell balance, but unaffected B cell capacity to limit monocyte activation in anti-neutrophil cytoplasmic antibody-associated vasculitis in remission. Rheumatology (Oxford) 2014;53:1683-92.

41. Todd S K, Pepper R J, Draibe J, Tanna A, Pusey C D, Mauric C, et al. Regulatory $B$ cells are numerically but not functionally deficient in anti-neutrophil cytoplasm antibody-associated vasculitis. Rheumatology 2014;53:1693-703.

42. Hu X, Tai J, Qu Z, Zhao S, Zhang L, Li M, et al. A lower proportion of regulatory $B$ cells in patients with Henoch-Schoenlein purpura nephritis. PLoS One. 2016;11:e0152368.

43. van der Geest KS, Abdulahad WH, Chalan P, Rutgers A, Horst G, Huitena MG, et al. Disturbed B cell homeostasis in newly diagnosed giant cell arteritis and polymyalgia rheumatica. Arthritis Rheumatol 2014;66:1927-38.

44. Li W, Tian X, Lu X, Peng Q, Shu X, Yang H, et al. Significant decrease in peripheral regulatory $B$ cells is an immunopathogenic feature of dermatomyositis. Sci Rep 2016;6:27479.

45. Sakkas L I, Chikanza I C, Platsoucas C D. Mechanisms of Disease: the role of immune cells in the pathogenesis of systemic sclerosis. Nat Clin Pract Rheumatol 2006;2:679-85.

46. Sakkas L I, Bogdanos D P. Systemic sclerosis: New evidence re-enforces the role of B cells. Autoimmun Rev 2016;15:155-61.

47. Mavropoulos A, Simopoulou T, Varna A, Liaskos C, Katsiari C G, Bogdanos D P, et al. Breg Cells Are Numerically Decreased and Functionally Impaired in Patients With Systemic Sclerosis. Arthritis Rheumatol 2016;68:494-504.

48. Matsushita T, Hamaguchi Y, Hasegawa M, Takehara K, Fujimoto $M$. Decreased levels of regulatory B cells in patients with systemic sclerosis: association with autoantibody production and disease activity. Rheumatology (Oxford) 2016;55:263-7.

49. Korniotis S, Gras C, Letscher H, Montandon R, Megret J, Siegert $\mathrm{S}$, et al. Treatment of ongoing autoimmune encephalomyelitis with activated B-cell progenitors maturing into regulatory B cells. Nat Commun 2016;7:12134.

50. Park M J, Kwok S K, Lee S H, Kim E K, Park S H, Cho M L. Adipose tissue-derived mesenchymal stem cells induce expansion of interleukin-10-producing regulatory B cells and ameliorate autoimmunity in a murine model of systemic lupus erythematosus. Cell Transplant 2015;24:2367-77. 